\title{
Lister strain vaccinia virus, a potential therapeutic vector targeting hypoxic tumours
}

\author{
CT Hiley ${ }^{1}$, M Yuan ${ }^{1}$, NR Lemoine ${ }^{1,2}$ and Y Wang ${ }^{1,2}$ \\ ${ }^{1}$ Centre for Molecular Oncology \& Imaging, Institute of Cancer, Barts and the London School of Medicine and Dentistry, Queen Mary \\ University of London, London, UK and 'Sino-British Research Center for Molecular Oncology, Zhengzhou University, Zhengzhou, \\ PR China
}

Hypoxia contributes to the aggressive and treatmentresistant phenotype of pancreatic ductal adenocarcinoma. Oncolytic vaccinia virus has potential as an anti-tumour agent, but the ability to lyse hypoxic tumour cells is vital for clinical efficacy. We hypothesized that unique aspects of the poxvirus life cycle would protect it from attenuation in hypoxic conditions. We characterized and compared the viral protein production, viral replication, cytotoxicity and transgene expression of Lister strain vaccinia virus in a panel of pancreatic cancer cell lines after exposure to normoxic or hypoxic conditions. Viral protein production was not affected by hypoxia, and high viral titres were

Keywords: human pancreatic cancer; vaccinia virus; hypoxia produced in both normoxic and hypoxic conditions. Interestingly, there was a 3.5-fold $(\mathrm{P}<0.001)$ and 20 -fold $(\mathrm{P}<0.0001)$ increase in viral cytotoxicity for CFPac1 and MiaPaca2 cell lines, respectively, in hypoxic conditions. Cytotoxicity was equivalent in the remaining cell lines. Levels of transgene expression (luciferase reporter gene) from the vaccinia viral vector were comparable, regardless of the ambient oxygen concentration. The present study suggests that the vaccinia virus is a promising vector for targeting pancreatic cancer and potentially other hypoxic tumour types. Gene Therapy (2010) 17, 281-287; doi:10.1038/gt.2009.132; published online 5 November 2009

\section{Introduction}

Solid tumours are characterized by regions of hypoxia that are inherently resistant to both radiotherapy and chemotherapy. ${ }^{1}$ Many studies have shown that a wide variety of tumour types exhibit hypoxia-related resistance mechanisms, resulting in a worse prognosis. ${ }^{2,3}$ Pancreatic ductal adenocarcinoma (PDAC) remains a disease with a dismal prognosis. The majority of patients present with metastatic disease, and attempts to alter the natural history with conventional chemotherapy have shown limited benefit. Response rates of only 5-20\% are seen and median survival for those with advanced disease remains less than six months. ${ }^{4}$ PDACs contain significant areas of hypoxia that have been measured intraoperatively in a clinical setting. ${ }^{5}$ In addition, hypoxia is associated with a poor prognosis in $\mathrm{PDAC}^{6}$ and is implicated in the resistance to gemcitabine, the current standard of care. ${ }^{7}$ Therefore, development of novel therapeutics to conquer this obstacle is pivotal to improve the survival from this lethal disease.

Replicating oncolytic viruses have a natural tropism for tumour cells. Further modification of viruses enables selective tumour targeting and offers the possibility of

Correspondence: Professor NR Lemoine or Dr Y Wang, Institute of Cancer, Barts \& The London School of Medicine, Queen Mary University of London, Charterhouse Square, London EC1 6BQ, UK. E-mail: Director@qmcr.qmul.ac.uk or yaohe.wang@qmul.ac.uk Received 13 June 2009; revised 7 September 2009; accepted 14 September 2009; published online 5 November 2009 treating cancers that are resistant to conventional therapies. The oncolytic viruses are not subject to the same resistance mechanisms as conventional cytotoxic therapies, and are effective even if apoptosis is blocked. ${ }^{8}$ Despite encouraging laboratory data, clinical trials using oncolytic viral therapy for pancreatic cancer have shown safety, but with limited efficacy. A replication-selective oncolytic adenovirus, Onyx 015 (dl1520), has been administered by intratumoral injection to patients with locally advanced pancreatic tumours in phase I/II trials. Although treatments were well tolerated, no objective responses were seen in patients after virus alone was administered, and only two of 21 patients showed objective responses when gemcitabine was used in combination. , $^{10}$

One major hurdle affecting oncolytic adenovirus potency is the tumour environment, which can affect different stages of the viral life cycle. Recent studies show that replication of Adenovirus serotype 5, the most commonly used oncolytic viral vector, is attenuated in hypoxic conditions. Expression of cell-surface receptors for adenovirus, Coxsackie/Adenovirus receptor and $\alpha_{\mathrm{v}}$ integrins, is unaffected by hypoxia, as is the mRNA expression of critical viral genes such as E1A and Hexon. However, translation of viral mRNA to protein is reduced, resulting in a 10-100-fold reduction in the yield of infectious virus particles. ${ }^{11,12}$ In addition, the group B adenoviruses, serotype 3 and 11, are attenuated in hypoxia with both reduced lytic potential and production of virus particles independent of viral receptor status or viral gene expression. ${ }^{13}$ Consequently, 
adenoviruses may not be the ideal vectors for tumours with significant hypoxic fractions such as PDAC.

Vaccinia virus is an alternative oncolytic virus and has some potential advantages over other viral vectors. Vaccinia is a DNA virus with an extensive safety profile in humans, as the virus has been used in millions of people for the World Health Organization smallpox eradication programme. ${ }^{14}$ In comparison to adenoviral vectors, the virion particle size and DNA organization of vaccinia virus allows insertion of multiple transgenes with less deleterious effects on subsequent viral DNA replication, virion packaging and dissemination. ${ }^{15}$ As an Orthopoxvirus, the life cycle of vaccinia virus is entirely located in the cytoplasm of infected host cells, in contrast to other viral vectors that replicate in the nucleus and rely on host transcription factors for DNA replication. ${ }^{16}$ In addition, the infectious virion is packaged with pretranscribed early viral gene mRNA and ATP, consequently, viral replication is initiated early after infection and the life cycle of vaccinia virus is shorter than other oncolytic viruses. ${ }^{17}$

We hypothesized that, unlike adenovirus, the unique features of vaccinia virus would mean that its inherent oncolytic potential would not be deleteriously affected under hypoxic conditions. This hypothesis was further supported by two recent reports. First, it was shown that the entry of mature vaccinia virions into host cells is accelerated by brief low-pH through an endosomal pathway. ${ }^{18}$ Second, the tumour microenviroment is known to be hypoxic, and genes involved in regulating intracellular $\mathrm{pH}$ are upregulated by hypoxia-inducible factor $($ Hif- $1 \alpha) .{ }^{19}$ Given that pancreatic cancer has been shown to be one of the most hypoxic tumours, ${ }^{5}$ we therefore investigated the effect of hypoxia on the life cycle of vaccinia virus using pancreatic cancer as a model and characterized vaccinia virus as an alternative vector targeting hypoxic tumour cells.

\section{Results}

\section{Hypoxia stabilizes Hif- $1 \alpha$ and induces nuclear translocation}

Hypoxia-inducible factor- $1 \alpha$ is the key protein mediating the response of cells to a hypoxic microenvironment. In the presence of oxygen, Hif- $1 \alpha$ is hydroxylated at specific proline residues, which results in its interaction with the Von Hippel-Lindau gene product and subsequent ubiquitination and degradation. ${ }^{20}$ In the absence of ambient oxygen, this degradation does not occur and subsequent nuclear localization results in the transcription of Hif- $1 \alpha$ target genes and cellular adaptation to hypoxia. Vaccinia virus is becoming an increasingly common vector for viral gene and oncolytic therapy. However, its ability to replicate in hypoxic conditions has not been reported to date. In the present study, hypoxic conditions were simulated with the use of a hypoxic incubator maintaining the ambient oxygen concentration at $1 \% \mathrm{pO}_{2}$. Immunoblotting for Hif- $1 \alpha$ in nuclear extracts of three pancreatic cancer cell lines, shown in Figure 1, showed that nuclear localization of this protein was observed only when cells were exposed to $1 \% \mathrm{pO}_{2}$ and validates the use of this technique in subsequent experiments. This suggests that the environment used in the present study is suitably hypoxic.

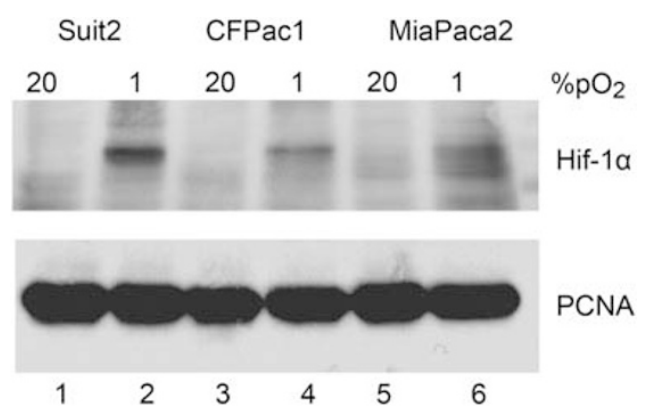

Figure 1 Stabilization and nuclear translocation of hypoxiainducible factor $1 \alpha$ (Hif- $1 \alpha)$ under hypoxic conditions. Cell lines were incubated in normoxic conditions $\left(20 \% \mathrm{pO}_{2}\right)$ (lanes 1,3 and 5) or hypoxia $\left(1 \% \mathrm{pO}_{2}\right)$ (lanes 2,4 and 6$)$ for $16 \mathrm{~h}$ before harvesting of nuclear extracts for immunoblotting. Lysates were probed for Hif- $1 \alpha$ and proliferating cell nuclear antigen (PCNA) expression as a loading control.

\section{Vaccinia virus protein expression is not affected by hypoxic conditions}

Given the fact that viral protein expression is the direct indicator of initiation of the viral life cycle and hypoxia has been shown to limit the total amount of protein synthesis, ${ }^{21}$ we first investigated whether production of viral proteins in pancreatic cancer cell lines exposed to hypoxia would be altered. CFPac1 and Miapaca2 cell lines were infected with Lister vaccine strain of vaccinia virus (VVLister) at an multiplicity of infection (MOI) $=1$ and cell lysates were harvested at $24 \mathrm{~h}, 48 \mathrm{~h}$ and $72 \mathrm{~h}$ after infection. Similar levels of vaccinia virus protein were present at $72 \mathrm{~h}$ when exposed to normoxic or hypoxic conditions, as shown in lanes 6 and 7 of Figure 2a. Immunoblotting for stabilization and nuclear translocation of Hif- $1 \alpha$ from MiaPaca2 lysates was performed to confirm that exposure to hypoxia had been adequate during the experiment (Figure $2 b$ ). This result confirms that critical viral proteins are translated efficiently in hypoxic conditions. This also implies that the steps of the vaccinia virus life cycle before viral gene expression, such as attachment and internalization etc., may not be affected by hypoxia.

\section{Vaccinia virus replication under hypoxic conditions}

The ability of replication-competent viruses to infect, multiply, lyse and then subsequently infect neighbouring cells is crucial for them to spread throughout a tumour. There has been concern that hypoxia may present a barrier to this. ${ }^{22}$ We investigated the replication of VVLister in pancreatic cancer cell lines, Suit-2, MiaPaca2 and CFPac1, when exposed to normoxic or hypoxic conditions before and after viral infection. Cells and supernatant were collected at 24, 48, 72 and $96 \mathrm{~h}$ postinfection. The number of plaque-forming units (pfu) per cell produced for each cell line in different conditions was determined using a TCID50 (50\% tissue culture infective dose) assay, as described in the Materials and Methods section. The levels of viral replication in MiaPaca-2 and CFPac1 cells are unaffected at any point by hypoxic conditions (Figure 3 ). Suit2 cells show a similar pattern at $24 \mathrm{~h}$ and $48 \mathrm{~h}$, producing a high titre of infectious viral particles in both hypoxic and normoxic conditions. At later time points, even higher titres are 
a Mock $V V L+24 h \quad V V L+48 h \quad V V L+72 h$

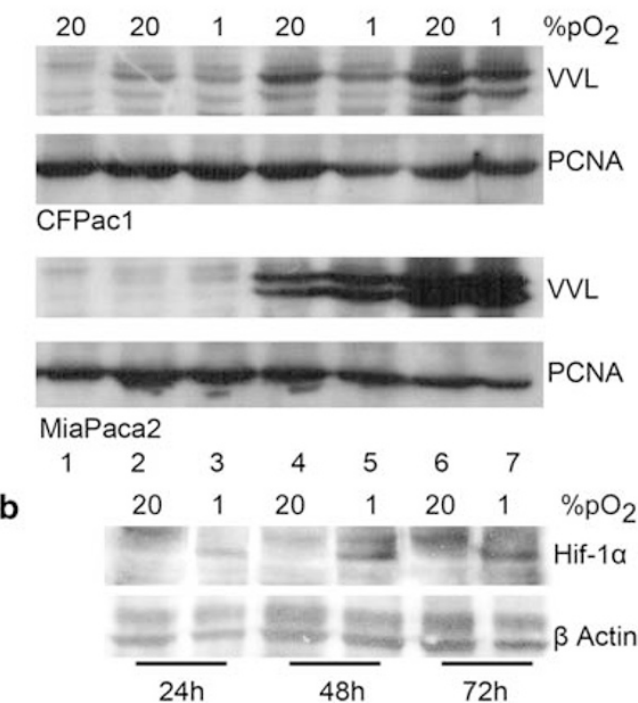

Figure 2 Viral gene expression of vaccinia virus in human pancreatic cancer after viral infection, and hypoxia-inducible factor $1 \alpha$ (Hif- $1 \alpha$ ) stabilization and nuclear translocation in normoxic or hypoxic cells. (a) Hypoxia does not affect viral gene expression of vaccinia virus. Cells were maintained in normoxic or hypoxic conditions before and after viral infection. Cells were infected with VVLister (Lister vaccine strain of vaccinia virus) at an multiplicity of infection $(\mathrm{MOI})=1$ or mock infected with vehicle buffer alone. Vaccinia virus protein was measured using an anti-vaccinia polyclonal antibody. Human proliferating cell nuclear antigen (PCNA) was used as a loading control; (b), Hif- $1 \alpha$ stabilization and nuclear translocation was shown on nuclear lysates from MiaPaca2 cells in normoxic $\left(20 \% \mathrm{pO}_{2}\right)$ or hypoxic cells $\left(1 \% \mathrm{pO}_{2}\right)$ over a time course. $\beta$-actin was used as a loading control.

achieved when replication occurs in ambient oxygen concentrations; however, there was no significant difference compared with those in hypoxia. In summary, high viral titres of VVLister (approximately more than $100 \mathrm{pfu}$ per cell) were achievable in all pancreatic cancer cell lines tested in both normoxic and hypoxic conditions.

\section{Enhanced cytotoxicity of vaccinia virus in hypoxia}

Effective lysis of infected tumour cells is the ultimate aim of oncolytic therapy. We used the MTS (3-(4, 5-dimethylthiazol-2-yl)-5-(3-carboxymethoxyphenyl)-2-(4sulfophenyl)-2H-tetrazolium) assay to determine the $\mathrm{EC}_{50}$ (dose of virus required to kill $50 \%$ of cells) for four pancreatic cancer cell lines. Cells were infected and maintained in the indicated oxygen conditions for the duration of the experiment and cell viability was analysed at 6 days post-infection. Dose-response curves and $\mathrm{EC}_{50}$ values were calculated, and the results were shown in Figure 4 and Table 1 . Cytotoxicity of vaccinia virus in Suit2 and Panc1 cell lines was maintained irrespective of a reduction in ambient oxygen concentration. Interestingly, for MiaPaca2 and CFPac1 cell lines, there was a statistically significant increase in vaccinia virus cytotoxicity in hypoxia with an $\sim 20$-fold $(P<0.0001)$ and 3.5 -fold $(P<0.001)$ reduction in $\mathrm{EC}_{50}$, respectively. These data suggest that VVLister is a potential agent for oncolytic virotherapy in which hypoxia occurs in the tumour microenvironment.
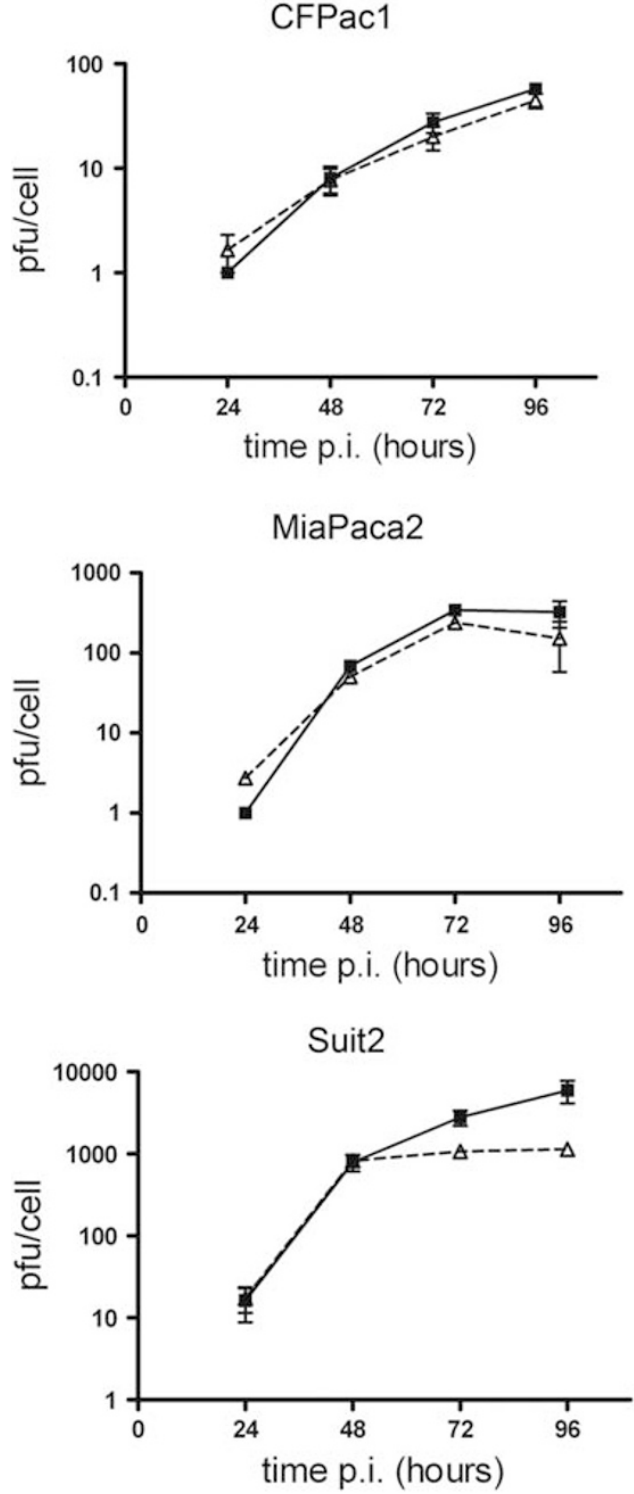

Figure 3 Viral replication of VVLister (Lister vaccine strain of vaccinia virus) in normoxic (solid line) and hypoxic conditions (dashed line) measured by TCID50 (50\% tissue culture infective dose) assay of viral burst assays. Cell lines were exposed to normoxic or hypoxic conditions before and after infection with a multiplicity of infection (MOI) $=1$ of VVLister. Burst assay samples were collected at $24,48,72$ and $96 \mathrm{~h}$ post-infection. TCID50 assays were performed on CV1 green monkey kidney cells. Experiments were performed in triplicate for each cell line, time point and condition. Results are presented as mean \pm standard deviation.

\section{Transgene expression in vaccinia virus vector is not affected by hypoxic conditions}

Many replicating viruses used for oncolytic therapy have additional therapeutic transgenes inserted into the viral genome to increase their therapeutic effect. Examples include pro-apoptotic proteins, prodrug-converting enzymes and cytokines. ${ }^{23}$ One of the attractions of vaccinia virus over alternative viral vectors is its large capacity for transgene insertion. ${ }^{17}$ Hypoxia will modulate the gene expression of any host cell; hence, verifying the effect of hypoxia on transgene expression from replication-competent vaccinia virus is important if this vector is to be of clinical use. ${ }^{21}$ We used VVL15 (a recombinant vaccinia 

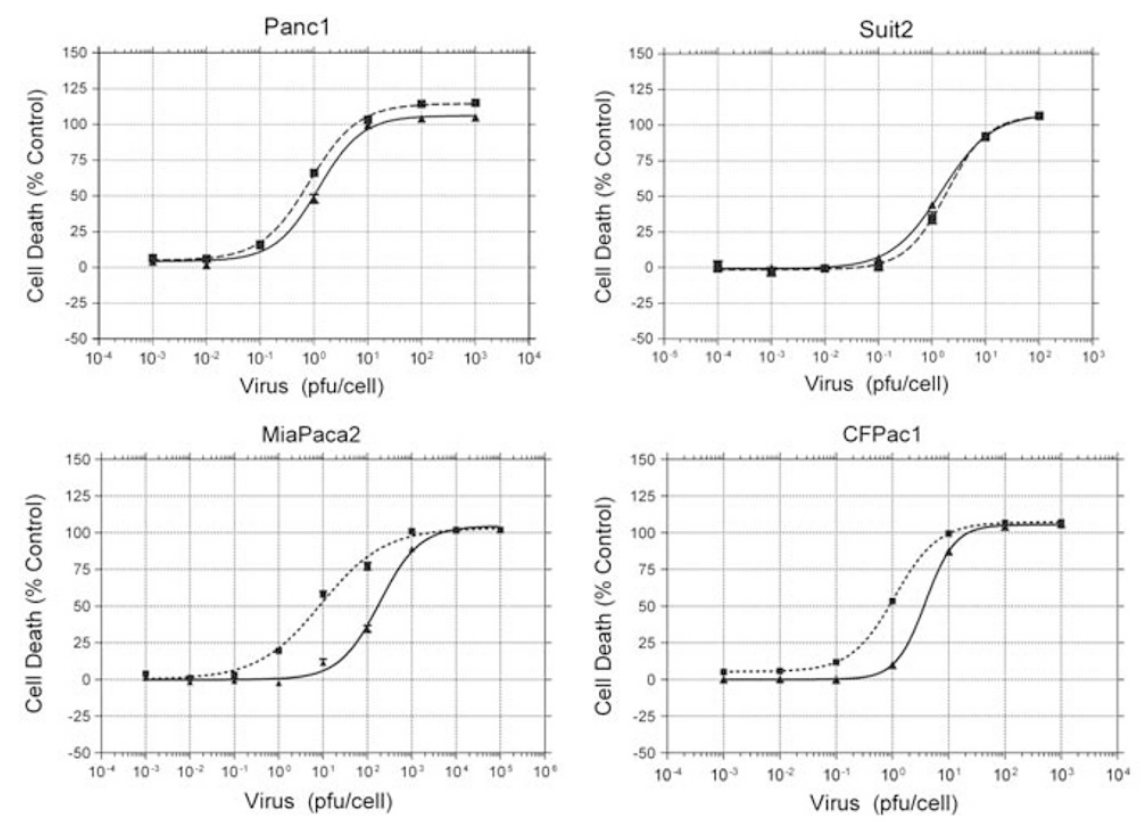

Figure 4 Effect of hypoxia on cytotoxicity of vaccinia virus Lister strain (VVLister). Pancreatic cancer cell lines were pre-treated with hypoxic or normoxic conditions for at least $16 \mathrm{~h}$, then infected with serial dilutions of VVLister and maintained under the same oxygen tension. The infected cells were assayed with MTS (3-(4,5-dimethylthiazol-2-yl)-5-(3-carboxymethoxyphenyl)-2-(4-sulfophenyl)-2Htetrazolium) reagent at day 6 post-infection at $37^{\circ} \mathrm{C}$ with $5 \% \mathrm{CO}_{2}$ for $2 \mathrm{~h}$. Viable cells were determined as a percentage of the noninfected controls and non-linear regression analysis was used to draw dose-response curves. Each assay contained six replicates and results are presented as mean \pm standard deviation of four independent experiments (dashed line: hypoxia, solid line: normoxia).

Table 1 Comparison of cytotoxicity of vaccinia virus in normoxia and hypoxia

\begin{tabular}{|c|c|c|c|c|c|}
\hline \multirow[t]{2}{*}{ Cell lines } & \multicolumn{2}{|c|}{$E C_{50}(95 \% C I)$} & \multirow[t]{2}{*}{ P-value } & \multicolumn{2}{|c|}{ Hill slope } \\
\hline & $20 \%$ Oxygen & $1 \%$ Oxygen & & $20 \%$ Oxygen & $1 \%$ Oxygen \\
\hline Panc1 & $1.21(1.03-1.41)$ & $0.82(0.71-0.95)$ & 0.07 & 1.075 & 0.9662 \\
\hline Suit2 & $1.50(1.24-1.81)$ & $1.88(1.37-2.59)$ & 0.48 & 0.924 & 1.12 \\
\hline MiaPaca2 & $187.00(154.39-226.37)$ & 9.15 (7.24-11.58) & $<0.00004$ & 0.9555 & 0.6065 \\
\hline CFPac1 & $3.89(3.59-4.20)$ & $1.10(1.04-1.16)$ & $<0.002$ & 1.66 & 1.122 \\
\hline
\end{tabular}

Abbreviations: $\mathrm{CI}$, confidence interval; $\mathrm{EC}_{50}$, the concentration of virus required to kill $50 \%$ of cells.

The $\mathrm{EC}_{50}$ values of vaccinia virus in the four different pancreatic cancer cell lines is presented with $95 \% \mathrm{CI}$ and $P$-values represent any significant difference between infection in normoxia versus hypoxia. Hill slope values for the dose-response curves are also presented.

virus derived from the VVLister) in which the firefly luciferase reporter gene was inserted into the thymidine kinase region downstream of the early-late vaccinia p7.5 promoter to assess transgene expression. The levels of luciferase activity after infection of four pancreatic cancer cell lines were detected by the Live Imaging System IVS200 (Xenogen, Alameda, CA, USA) at multiple time points, as opposed to isolated readings or early time points, as used in other studies to produce more representative data on gene expression. ${ }^{24,25}$ As shown in Figure 5, luciferase expression was largely unaffected by hypoxia. Only two of the four cell lines tested showed a significant difference between normoxic and hypoxic cells at two isolated time points. There was a decrease at $24 \mathrm{~h}$ and increase at $48 \mathrm{~h}$ in luciferase expression for CFPac1 and Panc1, respectively. However, this difference was not sustained at later time points. This result suggests that hypoxia does not compromise transgene expression from replication-competent vaccinia virus.

\section{Discussion}

Wild-type vaccinia virus has been well characterized, and much data on the molecular biology, genome sequence, viral life cycle and immunology have been reported. Vaccinia virus is an appealing candidate agent for oncolytic virotherapy because of these inherent properties. Besides several other attractive qualities (such as fast and efficient replication with rapid cell-tocell spread; natural tropism for tumours; strong lytic ability; large cloning capacity; well-defined molecular biology; safety in human beings; and good stability ${ }^{26-29}$ ), a defining feature that we showed in the present study is that hypoxia does not significantly affect viral gene expression, viral replication, cytotoxicity and even enhances the tumour-killing activity in some tumour cell lines.

We have shown here that VVLister shows comparable efficacy in infection, replication and transgene expression, 

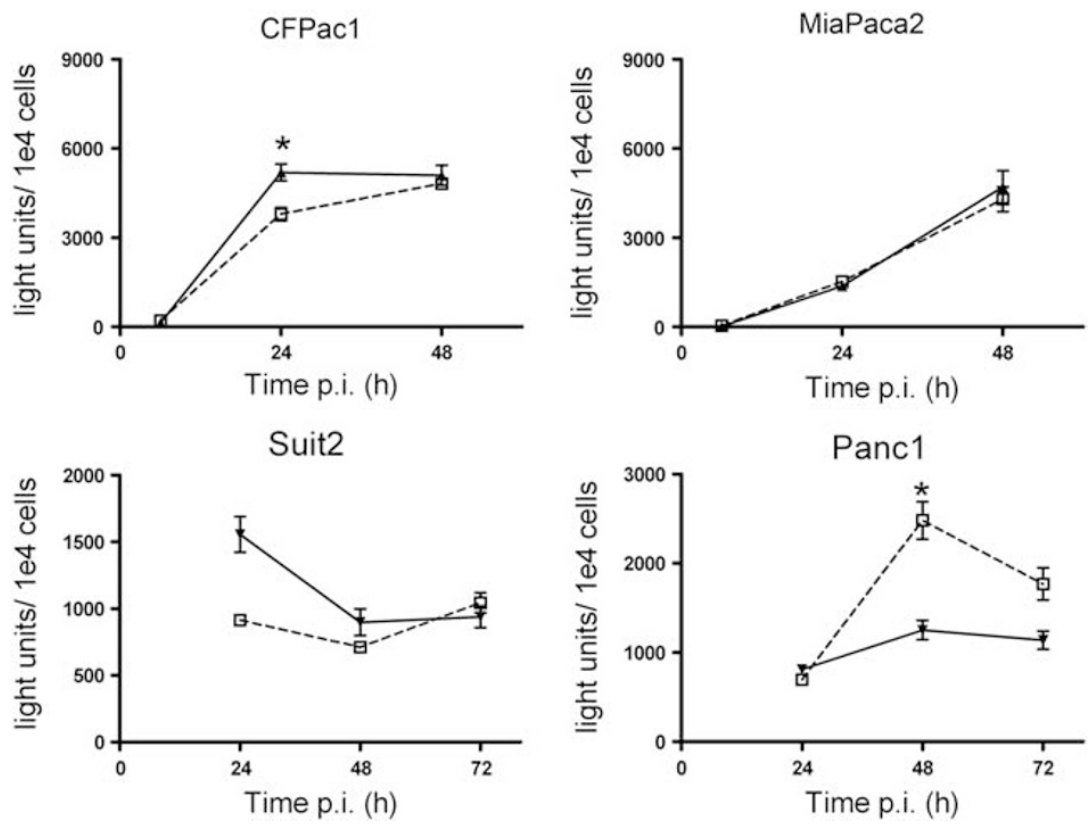

Figure 5 The effect of hypoxia on transgene expression from VVL15. Cells were infected with 1 pfu per cell of VVL15 and luciferase activity was measured at the time points indicated. All experiments were performed in triplicate and results represent the data from three separate experiments. Results are presented as mean \pm s.d. (solid line and triangle $=20 \% \mathrm{pO}_{2}$, dashed line and square $=1 \% \mathrm{pO}$. Light units, photons per second per $\left.\mathrm{cm}^{2} .{ }^{*} P<0.05\right)$. pfu, plaque-forming unit.

regardless of the ambient oxygen concentration. It is important to highlight that hypoxic cells used in these experiments had been exposed to reduced oxygen concentration for at least $16 \mathrm{~h}$ before infection, which is more likely to reflect cellular adaptation to hypoxia and model clinical vaccinia virus infection than shorter exposure times that have been used for the study of other oncolytic viruses. ${ }^{12,24}$ In addition, we found that effective tumour cell lysis was maintained after infection of hypoxic PDAC cell lines, and in half of the cell lines tested, there was a statistically significant improvement in viral cytotoxicty. This is an important result given that tumour lysis is the ultimate goal of oncolytic therapy.

Many groups have tried to target hypoxic fractions of tumours using hypoxia-specific promoters often containing hypoxia-response elements that facilitate Hif- $1 \alpha$ binding and downstream gene transcription. Unfortunately, such promoters are invariably less powerful drivers of gene expression than constitutive viral promoters, and result in lower levels of gene expression and viral replication relative to wild-type viruses. ${ }^{30,31}$ Our results show that vaccinia virus has the capacity to infect and replicate in hypoxic tumour cells without the need for such approaches. A recent report from Connor et al. ${ }^{24}$ showed that oncolytic vesicular stomatitis virus has comparable viral replication in normoxic HeLa cells compared with those exposed to $1 \% \mathrm{O}_{2}$ for $2 \mathrm{~h}$ before infection and only a slight reduction of viral-induced cytopathic effect was seen on semi-quantitative analysis. The availability of other oncolytic viruses that are not significantly attenuated in hypoxia in comparison with adenoviral strains is a welcome finding. However, our results suggest a several-fold, statistically significant improvement in the oncolytic potential of vaccinia virus in some pancreatic cell lines exposed to hypoxic conditions. The underlying mechanisms are not clear, which warrants further investigation. Connor et al. ${ }^{24}$ conclude that their results suggest an advantage of RNA viruses over DNA viruses in targeting hypoxic tumour cells because of a greater reduction of DNA synthesis in hypoxic cells. Vaccinia virus is a double-stranded DNA virus and similar to vesicular stomatitis virus replicates in the cytoplasm, encodes its own polymerases and is consequently less dependent on host gene and protein expression. Our alternative conclusion is that it is a cytoplasmic life cycle, with a reduced dependence on host gene and protein expression, rather than the nucleic acid construction of the viral genome that dictates the efficacy of oncolytic viruses in hypoxia.

In summary, we report the comparable efficacy of oncolytic vaccinia virus (including direct cancer cell killing and transgene expression) in both normoxic and hypoxic pancreatic tumour cells. These results suggest that vaccinia virus may be a potent therapeutic vector for targeting pancreatic cancer and potentially other hypoxic tumour types.

\section{Materials and methods}

\section{Cells and tissue culture conditions}

All cell lines were obtained from Cancer Research UK Central Cell Services and maintained in Dulbecco's modified Eagle's medium containing $0.06 \mu \mathrm{g}^{-1}$ penicillin and $0.1 \mu \mathrm{g} \mathrm{l}^{-1}$ streptomycin with $10 \%$ fetal calf serum. Cell lines were cultured at $37{ }^{\circ} \mathrm{C}$ with $5 \% \quad \mathrm{CO}_{2}$ unless otherwise specified. Similarly, low passage numbers were used for each experiment.

\section{Viral stocks and viral infection}

The Lister vaccine strain of vaccinia virus (VVLister) and the recombinant luciferase-expressing vaccinia viruses 
(VVL15) were constructed and kindly provided by Professor Istvan Fodor (Loma Linda University Campus, Loma Linda, CA, USA). VVL15 was constructed by the insertion of the firefly luciferase and the lac $Z$ reporter genes into the thymidine kinase region of VVLister downstream of the early-late vaccinia p7.5 promoter. $^{32}$ Cells were trypsinized, replated and exposed to either normoxic or hypoxic conditions for $16 \mathrm{~h}$ before any viral infection. Infections were performed using a $\mathrm{MOI}=1 \mathrm{pfu}$ per cell unless otherwise specified.

\section{Hypoxia}

For this study, hypoxia is defined as $1 \%$ oxygen, which is an oxygen concentration of $\sim 7 \mathrm{~mm} \mathrm{Hg}$. This was achieved using a hypoxic incubator maintained at $94 \%$ nitrogen, $5 \% \quad \mathrm{CO}_{2}$ and $1 \%$ oxygen (Heto-Holten Cell Chamber 170, Surrey, UK).

\section{Immunoblotting}

Nuclear extracts were isolated using the NE-PER nuclear and cytoplasmic extraction reagents (Pierce, Rockford, IL, USA) according to the manufacturer's instructions. Whole cell lysates were prepared by removing adherent cells using a cell scraper, washing cells in $1 \mathrm{ml}$ of phosphate-buffered saline at $4{ }^{\circ} \mathrm{C}$ and centrifuging at 2000 r.p.m. for $5 \mathrm{~min}$ at $4{ }^{\circ} \mathrm{C}$. After the removal of phosphate-buffered saline, cells were resuspended in $50 \mu \mathrm{l}$ of NP40 cell lysis buffer (50 mM Tris, pH7.4, $150 \mathrm{mM}$ $\mathrm{NaCl}, 10 \mathrm{mM} \mathrm{Ca}{ }^{2+}$ ), protease inhibitor cocktail (Roche Applied Science, Mannheim, Germany) and 1\% Nonidet P40 (Sigma Chemicals Co., Poole, UK) before storage at $-80{ }^{\circ} \mathrm{C}$. Total protein concentration was determined using the BCA protein assay (Pierce), and equal amounts of protein were electrophoresed on a reducing denaturing $10 \%$ polyacrylamide gel. Proteins were transferred by electroblotting to a polyvinylidene fluoride membrane (Immobilon-P, Millipore, Bedford, MA, USA). Non-specific binding was blocked using $5 \%$ bovine serum albumin in phosphate-buffered saline-Tween 20 (0.1\% Tween-20). Antibodies were incubated in $3 \%$ bovine serum albumin in phosphate-buffered saline-Tween 20. The murine monoclonal Hif- $1 \alpha$ antibody (AbCam Plc, Cambridge, UK) was used at a dilution of 1:750. The rabbit polyclonal vaccinia virus coat protein antibody (MorphoSys UK Ltd, Bath, UK) was used at a dilution of 1:1000. The PCNA (proliferating cell nuclear antigen) antibody (Santa Cruz Biotech Inc, Santa Cruz, CA, USA) was used at a dilution of 1:1000. The $\beta$-actin antibody was used at a dilution of 1:3000 (AbCam Plc). The appropriate anti-mouse or anti-rabbit secondary antibody (Santa Cruz Biotech Inc) was used at a dilution of 1:1000. Chemiluminescent detection was performed using ECL detection reagent (GE Healthcare, Buckinghamshire, UK) according to the manufacturer's instructions.

\section{Vaccinia virus replication assay}

Cells were seeded in triplicate at a density of $2 \times 10^{5}$ cells. All plates were incubated overnight in normoxic or hypoxic conditions. Plates were infected $16 \mathrm{~h}$ later with VVLister at a MOI $=1 \mathrm{pfu}$ per cell. Cells and supernatant were harvested using a cell scraper. Samples were freezethawed thrice and then centrifuged. The viral titre in each sample was determined by measuring the TCID $_{50}$ on indicator CV1 green monkey kidney cells. The cytopathic effect on CV1 was determined by light microscopy 10 days after infection. The Reed-Muench accumulate method was used to calculate the TCID50 value for each sample. ${ }^{33}$ Triplicates were used for each time point and each replicate had cytopathic effect assayed twice. Viral burst titres were converted to pfu per cell based on the number of cells present at viral infection.

\section{MTS Assay}

Cells were seeded in medium supplemented with 5\% fetal calf serum in 96-well plates, and maintained under hypoxic or normoxic conditions for $16 \mathrm{~h}$ before infection. On the day of infection, medium containing serial dilutions of vaccinia virus was added to each well. Cell viability was measured at 6 days by the MTS assay to assess vaccinia virus cytotoxicity. The MTS assays were carried out by adding $20 \mu \mathrm{l}$ of the reagent provided in CellTiter96 Aqueous Nonradioactive cell proliferation assay kit (Promega, Madison, WI, USA) to each well and the plates were incubated at $37{ }^{\circ} \mathrm{C}$ with $5 \% \mathrm{CO}_{2}$ for $2-3 \mathrm{~h}$. Hypoxic plates were always incubated in the hypoxic chamber for the entire duration of the experiment. Cell viability was determined by measuring the absorbance or optical density at $490 \mathrm{~nm}$ using an Opsys MR 96-well plate absorbance reader (Dynex, Chantilly, VA, USA), and a dose-response curve created by non-linear regression using Prism (GraphPad Software, La Jolla, CA, USA), allowing calculation of $\mathrm{EC}_{50}$ for each cell line and oxygen condition. The cell viability was measured as a percentage of viable cells remaining in the infected wells against viable cells remaining in the non-infected wells. Each assay contained six replicates and each assay was repeated four times.

\section{Reporter gene expression detected by IVIS camera}

in vitro

Cells were infected with VVL15 for 24,48 or $72 \mathrm{~h}$ and the luciferase activity measured using an IVIS camera (In Vivo Imaging System; Xenogen Corp., Alameda, CA, USA). Approximately $5 \times 10^{4}$ cells were seeded in $0.5 \mathrm{ml}$ of media with $10 \%$ fetal calf serum in 24 -well plates. Cells were incubated in normoxic or hypoxic conditions as indicated for $16 \mathrm{~h}$. Cells were harvested from control plates and the mean number of cells per well was used to calculate the amount of virus required for infection. Cells were infected with a MOI of 1 pfu per cell of VVL15 in Dulbecco's modified Eagle's medium with $5 \%$ fetal calf serum. At 24,48 and $72 \mathrm{~h}$ after infection, luciferase expression was determined as per the manufacturer's instructions. Media was replaced with $150 \mu \mathrm{g} \mathrm{ml}^{-1} \mathrm{D}$-luciferin (Xenogen Corp.) in serumfree medium at $37{ }^{\circ} \mathrm{C}$ and luminescence was measured two minutes later. Light emission was quantified as the sum of all detected photon counts within uniform-sized regions of interest with each well manually defined during post-data acquisition image analysis. This was measured in photons per second per $\mathrm{cm}^{2}\left(\mathrm{p} \mathrm{s}^{-1} \mathrm{~cm}^{-2}\right)$ using Living Image software (Xenogen Corp.). The mean light emission per cell ( $\mathrm{p} \mathrm{sec}^{-1} \mathrm{~cm}^{-2}$ per cell) was calculated using the number of cells infected at time 0 and compared for each MOI at 24, 48 and $72 \mathrm{~h}$. 


\section{Statistical analysis}

The unpaired students $t$-test was used for all statistical analysis unless otherwise specified.

\section{Conflict of interest}

The authors declare no conflict of interest.

\section{Acknowledgements}

This project is supported by Cancer Research UK (C633A6253/A6251), and Barts and The London Research Advisory Board. We are very grateful to Professor Istvan Fodor of Loma Linda University, Loma Linda, CA, USA, for providing the viruses.

\section{References}

1 Harris AL. Hypoxia-a key regulatory factor in tumour growth. Nat Rev Cancer 2002; 2: 38-47.

2 Hiley CT, Green MML, Shanks JH, Bottomley IC, West CML, Cowan RA et al. Expression of vascular endothelial growth factor (VEGF) in locally invasive prostate cancer is prognostic for radiotherapy outcome. Int J Radiat Oncol Biol Phys 2007; 67: 84-90.

3 Hutchison GJ, Valentine HR, Loncaster JA, Davidson SE, Hunter RD, Roberts SA et al. Hypoxia-inducible factor 1alpha expression as an intrinsic marker of hypoxia: correlation with tumor oxygen, pimonidazole measurements, and outcome in locally advanced carcinoma of the cervix. Clin Cancer Res 2004; 10: $8405-8412$

4 Ghaneh P, Costello E, Neoptolemos JP. Biology and management of pancreatic cancer. Postgrad Med J 2008; 84: 478-497.

5 Koong AC, Mehta VK, Le QT, Fisher GA, Terris DJ, Brown JM et al. Pancreatic tumors show high levels of hypoxia. Int J Radiat Oncol Biol Phys 2000; 48: 919-922.

6 Sun HC, Qiu ZJ, Liu J, Sun J, Jiang T, Huang KJ et al. Expression of hypoxia-inducible factor-1 alpha and associated proteins in pancreatic ductal adenocarcinoma and their impact on prognosis. Int J Oncol 2007; 30: 1359-1367.

7 Yokoi K, Fidler IJ. Hypoxia increases resistance of human pancreatic cancer cells to apoptosis induced by gemcitabine. Clin Cancer Res 2004; 10: 2299-2306.

8 Hawkins LK, Lemoine NR, Kirn D. Oncolytic biotherapy: a novel therapeutic platform. Lancet Oncol 2002; 3: 17-26.

9 Mulvihill S, Warren R, Venook A, Adler A, Randlev B, Heise C et al. Safety and feasibility of injection with an E1B-55 kDa genedeleted, replication-selective adenovirus (ONYX-015) into primary carcinomas of the pancreas: a phase I trial. Gene Therapy 2001; 8: 308-315.

10 Hecht JR, Bedford R, Abbruzzese JL, Lahoti S, Reid TR, Soetikno RM et al. A phase I/II trial of intratumoral endoscopic ultrasound injection of ONYX-015 with intravenous gemcitabine in unresectable pancreatic carcinoma. Clin Cancer Res 2003; 9: 555-561.

11 Pipiya T, Sauthoff H, Huang YQ, Chang B, Cheng J, Heitner S et al. Hypoxia reduces adenoviral replication in cancer cells by downregulation of viral protein expression. Gene Therapy 2005; 12: 911-917.

12 Shen B, Hermiston T. Effect of hypoxia on Ad5 infection, transgene expression and replication. Gene Therapy 2005; 12: 902-910.
13 Shen BH, Bauzon M, Hermiston TW. The effect of hypoxia on the uptake, replication and lytic potential of group $B$ adenovirus type 3 (Ad3) and type 11p (Ad11p). Gene Therapy 2006; 13: 986-990.

14 Fenner F. Smallpox and Its Eradication (History of International Public Health, No. 6). World Health Organization: Geneva, 1988.

15 Smith GL, Moss B. Infectious poxvirus vectors have capacity for at least 25000 base pairs of foreign DNA. Gene 1983; 25: 21-28.

16 Schramm B, Locker J. Cytoplasmic organization of POXvirus DNA replication. Traffic 2005; 6: 839-846.

17 Thorne SH. Oncolytic vaccinia virus: from bedside to benchtop and back. Curr Opin Mol Ther 2008; 10: 387-392.

18 Townsley A, Weisberg A, Wagenaar T, Moss B. Vaccinia virus entry into cells via a low-pH-dependent endosomal pathway. J Virol 2006; 80: 8899-8908.

19 Swietach P, Vaughan-Jones RD, Harris AL. Regulation of tumor $\mathrm{pH}$ and the role of carbonic anhydrase 9. Cancer Metastasis Rev 2007; 26: 299-310.

20 Masson N, Ratcliffe PJ. HIF prolyl and asparaginyl hydroxylases in the biological response to intracellular $\mathrm{O}(2)$ levels. J Cell Sci 2003; 116: 3041-3049.

21 Kraggerud SM, Sandvik JA, Pettersen EO. Regulation of protein synthesis in human cells exposed to extreme hypoxia. Anticancer Res 1995; 15: 683-686.

22 Hay JG. The potential impact of hypoxia on the success of oncolytic virotherapy. Curr Opin Mol Ther 2005; 7: 353-358.

23 Bhattacharyya M, Lemoine NR. Gene therapy developments for pancreatic cancer. Best Pract Res Clin Gastroenterol 2006; 20: 285-298.

24 Connor JH, Naczki C, Koumenis C, Lyles DS. Replication and cytopathic effect of oncolytic vesicular stomatitis virus in hypoxic tumor cells in vitro and in vivo. J Virol 2004; 78: 8960-8970.

25 Shen B, Bauzon M, Hermiston T. The effect of hypoxia on the uptake, replication and lytic potential of group $B$ adenovirus type 3 (Ad3) and type 11p (Ad11p). Gene Therapy 2006; 13: 986-990.

26 Poland GA, Grabenstein JD, Neff JM. The US smallpox vaccination program: a review of a large modern era smallpox vaccination implementation program. Vaccine 2005; 23: 2078-2081.

27 Roenigk Jr HH, Deodhar S, St Jacques R, Burdick K. Immunotherapy of malignant melanoma with vaccinia virus. Arch Dermatol 1974; 109: 668-673.

28 Kim JH, Oh JY, Park BH, Lee DE, Kim JS, Park HE et al. Systemic armed oncolytic and immunologic therapy for cancer with JX-594, a targeted poxvirus expressing GM-CSF. Mol Ther 2006; 14: 361-370.

29 Park BH, Hwang T, Liu TC, Sze DY, Kim JS, Kwon HC et al. Use of a targeted oncolytic poxvirus, JX-594, in patients with refractory primary or metastatic liver cancer: a phase I trial. Lancet Oncol 2008; 9: 533-542.

30 Binley K, Iqball S, Kingsman A, Kingsman S, Naylor S. An adenoviral vector regulated by hypoxia for the treatment of ischaemic disease and cancer. Gene Therapy 1999; 6: 1721-1727.

31 Binley K, Askham Z, Martin L, Spearman H, Day D, Kingsman S et al. Hypoxia-mediated tumour targeting. Gene Therapy 2003; 10: 540-549.

32 Hung CF, Tsai YC, He L, Coukos G, Fodor I, Qin L et al. Vaccinia virus preferentially infects and controls human and murine ovarian tumors in mice. Gene Therapy 2007; 14: 20-29.

33 Reed LJ, Muench H. A simple method of estimating fifty percent endpoints. Am J Hyg 1938; 27: 493-497. 